\title{
Physical activity among older people with sight loss
}

Published by Thomas Pocklington Trust

June 2014

Research to explore participation in physical activity among older people with sight loss was carried out for Thomas Pocklington Trust by Cassandra Phoenix and Meridith Griffin, University of Exeter Medical School, and Brett Smith and P. David Howe, Loughborough University.

The findings tell us about the benefits older people with visual impairment experience in being active and the challenges they face, and identify ways to increase participation.

\section{Summary findings}

- Older people with sight loss who were physically active described many motivating factors for being so, citing physical, mental, and emotional rewards of participation. Engaging in activities often offered the opportunity for social interaction, and brought immense pleasure.

- Social support, accessible transport, confidence and access to information about opportunities enabled people to be physically active.

- There were multiple, overlapping barriers for those who struggled to be active or more active. The barriers were linked to health, for example levels of physical fitness, co-existing health conditions and mental and emotional health. They also included transport availability and cost; variety and sustainability of opportunities; lack of social support; fears about safety and low confidence.

- Many aspects of leisure facilities, both inside and outside of buildings, and the natural environment, can act as barriers to physical activity. Good lighting and signage, and the removal of unnecessary and inconsistently placed obstacles in changing rooms and locker areas, make the built environment more accessible. 
- Older people with sight loss are not a homogenous group, and need a variety of opportunities to participate in physical activity. Preferences for style, format and level of participation will depend on previous physical activity involvement and experiences.

\section{Background}

Physically active older people have lower risk of ill-health including dementia and have higher levels of physical and cognitive function, psychological well-being and independence than inactive older people. However, one in nine people aged 60 and over in the UK are currently living with sight loss, and the health and well-being of this growing and ageing population requires attention. Research shows that visually impaired older people have poorer general health than those with good sight. Because visual impairment limits activity it also puts people at risk of developing additional medical conditions.

No systematic research exists concerning physical activity among older people with sight loss. To date both research and policy have had little to say about the involvement of older people who are experiencing sight loss in later life, nor how their participation in physical activity can be encouraged. Knowledge of this specific group is needed in order to make meaningful changes to activity levels and improved health and well-being.

\section{Research aim}

This research aimed to increase the knowledge base relating to physical activity among older people who are living with sight loss by exploring individual experiences of, and identifying barriers and aids to, participating in physical activity.

\section{Research methods}

Face-to-face interviews were conducted with 48 people with sight loss aged 60 to 97 . They were recruited in equal numbers from the South West of England, the Midlands, and London. The group was diverse with respect to age, gender, ethnicity, economic, marital and health status, and type and level of sight loss. Participants engaged in a range of physical activities, such as rifle-shooting, dance, walking, going to the gym, lawn bowls 
and swimming. Several people who defined themselves as 'inactive' were also included. Mobile interviews, where the researcher travelled or participated alongside the interviewee, and audio diaries were also completed with a small number of people in each location. Case studies of 'promising practice' emerging from the research were also compiled.

\section{Findings}

Two central themes emerged of factors that were facilitators or barriers to participating in physical activity: people's health, and accessibility and opportunity.

\section{Health}

Everyone interviewed linked physical activity to health; their own health and fitness was an incentive, a motivator or a barrier to participation.

\section{- Physical fitness}

Several participants listed the physical benefits they considered to be the result of participation in their chosen activity. For these people, outcomes such as weight loss and improved cardiovascular functioning were markers of their progress, allowing them to carry out everyday tasks with ease. At the other end of the spectrum were those who described themselves as lacking physical fitness, with inactivity being the main reason.

\section{- Activity loss}

Every participant regretted some activity loss that resulted from their visual impairment. With respect to physical activities, several participants explained that the first thing to go was any form of ball sport participation. Other physical activities began to carry significant risks due to impaired vision or the need to be able to judge obstacles or distances.

"I used to cycle a lot on the roads. I think that would now be most unwise. I might ride on a path, but I find riding on the roads now impossible, so I've given that up."

For some, participating in an activity was possible with support from a partner or friend, but if that was no longer available, participation would often cease.

Participants whose sight loss was more severe or sudden, talked about a loss of general physical abilities related to mobility including walking.

"I really don't do much at all. I'm not fit enough, and I can't see well enough. I've found my parameters, and I don't feel I can do any more, or go any further than that." 


\section{- Other health problems}

Coping with multiple health problems, such as poor circulation and joints, alongside sight loss, also had a negative impact on some people's capacity to walk and their general mobility. In contrast, many participants described using physical activity as part of their broader health care regimen, particularly to help control other chronic conditions.

\section{- Mental and emotional health}

Going beyond physical health, the majority of participants acknowledged the link between physical activity and mental and emotional health. For some older people with sight loss, participating in physical activity, whether in an organised group or not, offered a means and a reason to 'get out of the house'.

For those who did participate in physical activity, solitary activities offered them a welcome opportunity to engage in selfreflection, contemplation and escape. Those who preferred group or team exercise cited enjoyment both of the activity and social interaction.

\section{- Challenge and independence}

Those participants who were enthusiastic about physical activity reported many reasons for this, such as personal challenge, learning or developing a physical skill, improving scores, or winning a competitive match. For other participants, the activity was secondary to other perceived benefits.

"I'm very conscious of my independence and I want to retain that. The cycling that I do makes my legs strong, more stable. So I'm more confident in getting around without help. I like to go out on my own and get to places, and maintain my independent lifestyle." For those who did not, or felt they could not, participate in physical activity, it was the loss of independent mobility that they missed most.

\section{Accessibility and opportunity}

Each participant reported issues relating to accessibility of physical activities. These were primarily practical or structural, but also included psychological considerations such as confidence. 


\section{- Transport availability and cost}

Reports of transport availability and cost varied by region and were most frequently a barrier to participation in the South West, where community transport and public transport links are relatively poor. Carrying out physical activity often relied on being driven to the location. Concessionary travel passes were praised, particularly within London. Those in the Midlands had mixed experiences, depending on how close they were to urban areas and available transport links.

\section{- Variety, sustainability and consistency of opportunities}

Several participants praised their local sight loss organisation for the variety of physical activity opportunities offered, while others were less pleased. Many of the latter regretted the inconsistency of opportunity, and referred to activities that were promised and then not delivered, or offered and then taken away.

"Archery they started once, never heard of it again. Tandem riding - I went on one ride and I've not heard anything since. Gardening started, and then it stopped. And that's worse than not having it at all, to be honest. It raises people up, and down they come again."

\section{- Marketing of opportunities}

Several participants expressed interest in participating in organised physical activities for visually impaired people, but explained that they were unsure of what opportunities existed and how to find out about them. Those who had participated in such activities recalled having proactively and persistently sought information about available opportunities in their area.

\section{- Social support}

Many participants reported not having the level of support from family and friends that they would have liked, or having lost support for various reasons, such as being widowed. This made it difficult, if not impossible, to participate in physical activity. Those who lived alone expressed a desire for companionship with an activity. Several participants described their participation in physical activity as only being possible because of the support of an individual or organisation.

"I've gone tandem cycling with the same friend - we celebrate 20 years of riding together next year. I've every confidence in him... We try to get out at least once a week, and in the summer we stop at a local pub sometimes. So that's nice - it's a real pleasure, actually." 


\section{- Confidence, fear and safety}

Many people felt they lacked confidence to participate in physical activity. This was often linked to a fear of injury or falling, and as a result of being out of their known environment. Participants expressed many additional fears: getting lost, looking silly, and being a target for crime. Confidence, fear, and safety concerns had implications not just for mobility, but also for the types of physical activity participants chose, or would consider choosing.

\section{- Time}

Many participants referred to how the pace of life had changed for them. They described having to accept that everything will take them longer and, as such, life had a different cadence to it, including aspects of mobility and participation in physical activities. Other participants were less accepting of the increased time that physical tasks and activities now required. These participants bemoaned the fact that it often took longer to get there and back than performing the activity itself.

\section{- Leisure facilities and the built and natural environment}

Several participants were members of local gyms and attended regularly, reporting positive experiences. However, many of these positive stories were coupled with reports of previously poor experiences. Participants recounted being turned away because of health and safety regulations, or being asked to pay additional fees on top of their membership to have a dedicated personal trainer at every gym session. Participants' experiences, both good and bad, strongly highlighted that there are many considerations in making a gym facility accessible. These include:

- accessible equipment

- allocated time slots to use facilities

- staff awareness and training

- lighting

- signage

- furniture and equipment being left in the same, familiar positions

- locker and changing room facilities

- positive attitude towards inclusiveness 
The environment outside of buildings and facilities also proved challenging, including: pavement quality and grade, the location of bollards, street signs and rubbish bins. Participants explained that navigating the built environment took mental as well as physical energy, and this could limit physical activity participation. Similarly, the natural environment posed many obstacles that restricted the type, level, and location of physical activity engaged in by participants.

\section{Conclusions and recommendations}

Older people with sight loss describe various barriers and factors which can aid their participation in physical activity. These have important implications for their wellbeing, quality of life and leisure pursuits. Given the growing proportion of older people experiencing and living with sight loss, it is essential that visual impairment is incorporated into the larger ageing agenda.

Because reported barriers to being active often overlapped, and since older people with sight loss are a varied group, change needs to take place on several levels:

- structural and environmental e.g. transport

- cultural e.g. awareness regarding sight loss and older age

- personal e.g. confidence-building.

The range of opportunities to participate in different forms and levels of physical activity also needs expanding. Previous physical activity involvement and experiences need to be considered. The whole range of benefits to be gained from participation needs to be included in health promotion messages, e.g. the benefits to physical, mental and emotional health as well as the pleasure gained from being active and social interactions. Individual stories of active older people with sight loss can highlight how people are enabled to be more physically active and are excellent examples of what has worked well for some and perhaps could work well for others.

Relevant service providers should try to provide a variety of options for participation in physical activity, within local sight loss organisations and in partnership with existing mainstream community activities. Collaboration is essential to pool resources and knowledge, and to ensure development and consistency of opportunity across the country. There is also a need to engage community and commercial stakeholders in order to widen accessibility and promote compliance to anti-discrimination laws.
"I used to belong to a rambling group but it got to the point where I thought, no, I can't do this anymore.

So I stopped.

Tree roots, steps, things like that. Because I can't see the ground, I don't know if it's sloped or going up. You don't half jar your back all the time, if you aren't expecting a change in ground level. I've been whacked in the face with branches." 


\section{Authors}

Drs Cassie Phoenix, Meridith Griffin, Brett Smith and

P. David Howe.

\section{How to obtain further information}

The full research report highlights identified barriers, facilitators, and motivators in greater detail and includes case studies of promising practice. The report is available from:

Thomas Pocklington Trust

Pier House

90 Strand on the Green

London W4 3NN

Telephone: 02089950880

Email: research@pocklington-trust.org.uk

Web: www.pocklington-trust.org.uk

In this publication, the terms 'visually impaired people', 'blind and partially sighted people' and 'people with sight loss' are used interchangeably. 
Thomas Pocklington Trust

Housing and support for people with sight loss

Pier House

90 Strand on the Green

London

W4 3NN

Tel: 02089950880

Email: info@pocklington-trust.org.uk

ISBN 978-1-906464-59-2

Web: www.pocklington-trust.org.uk

Published by Thomas Pocklington Trust

Registered Charity No. 1113729

Company Registered No. 5359336

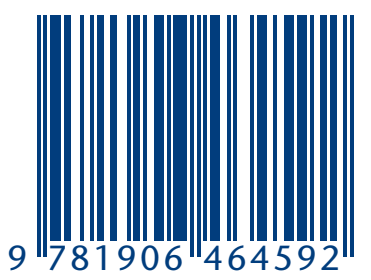

\section{www.pocklington-trust.org.uk}

